La structure interne de la Terre cache encore de nombreux

secrets que les géophysiciens tentent de percer.

Afin de pouvoir collecter des informations sur les couches les plus profondes du Globe les plus difficiles d'accès,

un nouvel outil est maintenant

au service de la géophysique :

le neutrino. Cette particule est émise lors des désintégrations $\beta$ d'éléments radioactifs, présents dans les couches terrestres et à l'origine d'une partie de la chaleur interne de la Terre.

La détection de ces neutrinos d'origine géologique, confirmée en 2015, peut permettre une compréhension plus détaillée du passé, du présent

et du futur de notre planète.

(a) Le flux de géoneutrinos émanant des désintégrations du potassium est plus intense d'un facteur 4 environ que celui provenant des chaines de désintégration de l'uranium et du thorium ; mais, à cause de leur faible énergie $(<1,5 \mathrm{MeV})$, leur détection étant hors de portée des détecteurs en opération, ces géoneutrinos ne sont pas pris en compte dans les bilans actuels.

\title{
Le neutrino au service de la géophysique
}

Vincent Fischer* (vfischer.physics@gmail.com) et Thierry Lasserre Service de Physique des Particules, Irfu, CEA/Saclay, 91191 Gif-sur-Yvette Cedex

* Adresse actuelle : Université de Californie à Davis (vfischer@ucdavis.edu)

\section{Le neutrino}

Le neutrino est la particule élémentaire la plus abondante de l'Univers. Cependant, il n'interagit que très peu avec la matière et sa détection est un défi pour les physiciens. Son existence, proposée de façon purement théorique par Wolfgang Pauli en 1930, fut finalement expérimentalement avérée en 1956 par une équipe de chercheurs américains dirigée par Clyde Cowan et Frederick Reines. Pendant les soixante dernières années, les innovations dans les domaines de la technologie des détecteurs et de l'analyse des données ont permis la détection des neutrinos émis par de nombreuses sources telles que les réacteurs nucléaires, le Soleil, les accélérateurs de particules et bien d'autres, entraînant la découverte de nombre de leurs propriétés. Il est désormais connu que le neutrino et son équivalent d'antimatière, l'antineutrino, sont présents dans la nature en trois types ou "saveurs ", chacune associée à un lepton élémentaire (électron, muon et tau). L'une de leurs propriétés les plus remarquables est leur capacité à se transformer entre ces différentes saveurs lors du phénomène d'oscillation, qui est d'origine purement quantique. Par exemple, un neutrino émis en tant que neutrino électronique peut ainsi se transformer en neutrino muonique lors de sa propagation. La découverte de ce phénomène, qui met en évidence l'existence d'une masse non nulle pour les neutrinos, par les expériences Super-Kamiokande au Japon et SNO au Canada, a été récompensée en 2015 par le prix Nobel de physique [1].

Alors que son application à la surveillance des réacteurs nucléaires a été récemment prouvée, le neutrino, ou plutôt l'antineutrino dans ce cas précis, devient un outil d'un intérêt particulier pour les sciences de la Terre. Les avancées expérimentales récentes dans la traque des antineutrinos résultant de la radioactivité bêta dans les chaînes de désintégrations de l'uranium, du thorium et du potassium 40 contenus dans la croûte et le manteau terrestre, promettent un avenir radieux à la détection de ces " géoneutrinos ", désormais un des objectifs avérés des physiciens des particules et des géophysiciens, avec pour ultime but d'améliorer la compréhension des entrailles de notre planète.

\section{Terre et géoneutrinos}

Le globe terrestre possède une structure en couches désormais bien caractérisée. La couche la plus externe, la croûte, d'une épaisseur d'environ $35 \mathrm{~km}$ sous les continents et $8 \mathrm{~km}$ sous les océans, entoure le manteau, qui se sépare en deux zones, supérieure et inférieure, la seconde s'étendant jusqu'à une profondeur de $2900 \mathrm{~km}$, au contact du noyau terrestre.

La composition chimique de chacune de ces couches est globalement connue. Alors que celle des parties externes, la croûte et le manteau supérieur, est accessible respectivement par l'analyse des roches issues de forages ou de remontées de lave, la composition des couches internes peut être extrapolée à partir de l'étude de météorites, dites chondrites carbonées, dont l'époque de formation dans le système solaire est similaire à celle de la Terre. Ces informations ont permis aux géophysiciens et géochimistes de développer certains modèles de la Terre, dits BSE ("Bulk Silicate Earth" en anglais, soit "Terre silicatée »), décrivant les concentrations des couches terrestres en silicium, oxygène et fer, les trois éléments les plus abondants. Cependant, d'importantes incertitudes, allant (en valeur relative) jusqu'à plusieurs dizaines de pourcents, demeurent sur la concentration en éléments radioactifs, communément appelés radioéléments. 


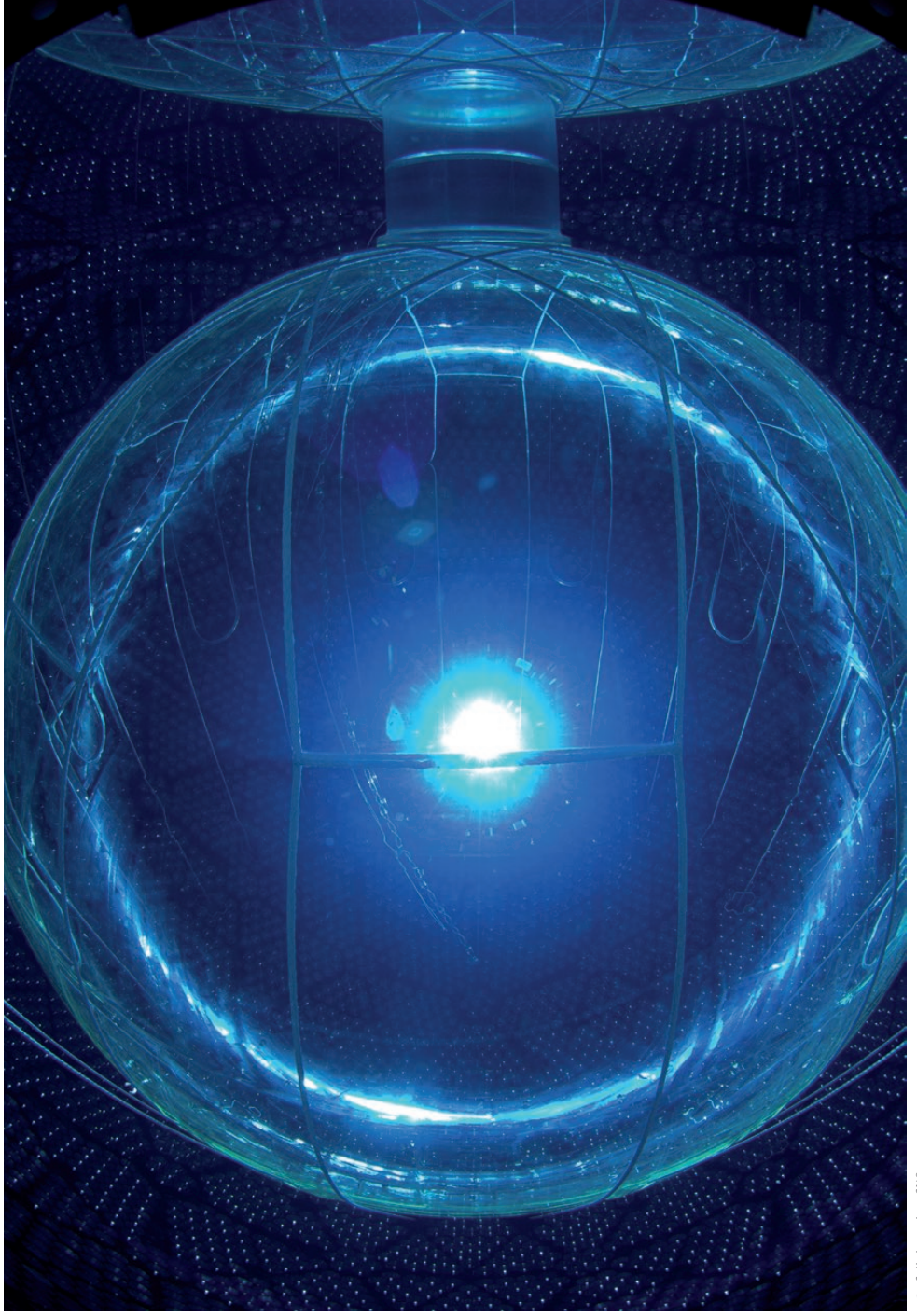

Photo de l'intérieur du détecteur souterrain de neutrinos SNO+ (Sudbury, Ontario, Canada), rempli d'eau ultra-pure.

Or, bien que leurs concentrations ne soient que de l'ordre de la partie par milliard, on estime qu'environ $50 \%$ de la chaleur interne dégagée par la Terre est induite par les désintégrations radioactives passées et présentes de ces éléments; l'autre moitié a pour origine les impacts de météorites à l'époque de la Terre primitive, ainsi que la contraction gravitationnelle. La dynamique interne de notre planète, dont dépendent la tectonique des plaques et le champ magnétique terrestre, est donc fortement affectée par la présence de ces radioéléments, dont les principaux sont des isotopes issus des désintégrations de l'uranium 238 $\left({ }^{238} \mathrm{U}\right)$ et du thorium $232\left({ }^{232} \mathrm{Th}\right)$, ainsi que le potassium $40\left({ }^{40} \mathrm{~K}\right)$.

Bien que, par le biais de nombreux forages à la surface du globe, le flux de chaleur surfacique terrestre ait été évalué à $47 \pm 2$ terawatts (TW) [2], la fraction de ce flux provenant des désintégrations successives de ces trois radioéléments, appelée chaleur radiogénique, demeure mal connue. Les trois principaux groupes de modèles BSE, géochimique, cosmochimique et géodynamique, chacun proposant des concentrations différentes en radioéléments, ne sont pas en accord dans leurs prédictions, parfois au sein du même groupe, et anticipent respectivement $20 \pm 4,11 \pm 2$ et $33 \pm 3$ TW de quantités de chaleur radiogénique, dont $6,8^{+1,4}{ }_{-1,1}$ dégagée par la croûte. Les radioéléments se concentrant de préférence dans les matériaux silicatés, leur présence dans le noyau terrestre est considérée comme négligeable. Par soustraction, le flux de chaleur dégagé par les radioéléments présents dans le manteau terrestre pourrait donc être compris dans une fourchette allant de 3 à 30 TW. D'où l'intérêt d'utiliser d'autres moyens que les forages pour obtenir une estimation plus précise de cette quantité, afin de trouver le modèle BSE représentant le plus fidèlement possible la mécanique interne de la Terre. Les radioéléments possèdent la caractéristique d'être instables et de se désintégrer en émettant de nombreuses particules, dont des neutrinos. Il est utile de faire la distinction entre le potassium, qui émet directement des neutrinos par désintégration bêta, et l'uranium et le thorium, qui se désintègrent par radioactivité alpha, et dont certains descendants émettent des neutrinos par radioactivité bêta. Alors que les autres sous-produits de ces désintégrations, principalement des électrons, des particules alpha et des rayons gamma, interagissent fortement avec la matière et contribuent ainsi à la génération de chaleur radiogénique, ces élusifs "géoneutrinos " parviennent à s'échapper des couches terrestres sans y interagir. Cependant, au vu de la masse importante de radioéléments dans la croûte et le manteau terrestre et grâce aux progrès instrumentaux et dans l'analyse des données, la détection de ces géoneutrinos est devenue possible, fournissant ainsi une nouvelle technique pour pouvoir sonder dans sa globalité l'inaccessible manteau.

Alors que la mesure du flux absolu de géoneutrinos donne une indication de l'activité radioactive de notre planète et donc du flux de chaleur radiogénique, la compréhension du spectre en énergie de ces neutrinos permettrait d'isoler les contributions des différents radioéléments. En effet, les descendants de l'uranium 238 et du thorium 232, ainsi que le potassium 40, émettent chacun des géoneutrinos d'énergies différentes et dont le spectre peut être mesuré de façon relativement précise par les détecteurs actuels dans les deux premiers cas. La mesure absolue du flux total de géoneutrinos, quant à elle, dépend directement du nombre d'événements observés dans les détecteurs. Les modèles BSE prédisent un flux total de géoneutrinos provenant des désintégrations de l'uranium et du thorium d'environ 4 millions par centimètre carré et par seconde ${ }^{(a)}$. Bien qu'élevé en apparence, ce flux est très inférieur aux 66 milliards de neutrinos solaires qui traversent la même surface dans le même intervalle de temps, et dont seulement une poignée est détectée chaque jour dans les détecteurs les plus performants actuellement en opération. 


\section{Techniques expérimentales}

Les antineutrinos émis par les désintégrations successives du thorium et de l'uranium étant de saveur électronique, leur détection s'effectue principalement à travers la réaction dite "bêta inverse " avec un noyau d'un atome d'hydrogène $\left(v+\right.$ proton $\rightarrow \mathrm{e}^{+}+$neutron $)$. La section efficace, que l'on peut traduire par la probabilité d'occurrence, de cette réaction est de plusieurs ordres de grandeur supérieure à celle des autres réactions de neutrinos aux mêmes énergies, ce qui en fait la plus prisée pour détecter des antineutrinos électroniques de basses énergies. Les détecteurs les plus sensibles à cette réaction, tel que KamLAND au Japon (fig. 1), sont dits "à liquide scintillant " et consistent le plus souvent en de grandes cuves remplies d'huiles minérales à base d'hydrocarbures, mélangées à des molécules aromatiques carbonées. Ces liquides organiques étant riches en noyaux d'hydrogène, ou protons libres, ils sont tout indiqués comme cibles de choix pour la réaction bêta inverse. Les produits de cette réaction, un positron et un neutron, vont déposer leur énergie dans le liquide qui, grâce à l'action des molécules aromatiques, va émettre de la lumière bleue et ultraviolette. Les deux impulsions lumineuses, corrélées en temps (de l'ordre de la centaine de microsecondes) et en distance (moins d'un mètre), et dont l'intensité est proportionnelle à l'énergie de la particule, sont finalement détectées par des photodétecteurs disposés sur les parois de la cuve.

À l'origine de nombreuses observations d'antineutrinos émis par des réacteurs nucléaires, des accélérateurs et même des explosions d'étoiles, cette méthode de détection a récemment permis aux physiciens de mettre en évidence le flux de géoneutrinos nous traversant à chaque instant. C'est ainsi qu'en juin 2015, l'expérience Borexino, dans le Laboratoire National du Gran Sasso en Italie centrale, fut la première à détecter des géoneutrinos avec une certitude de plus de cinq déviations standard, signe d'une découverte en physique des particules. Cette découverte est le fruit d'une traque de plus de quinze

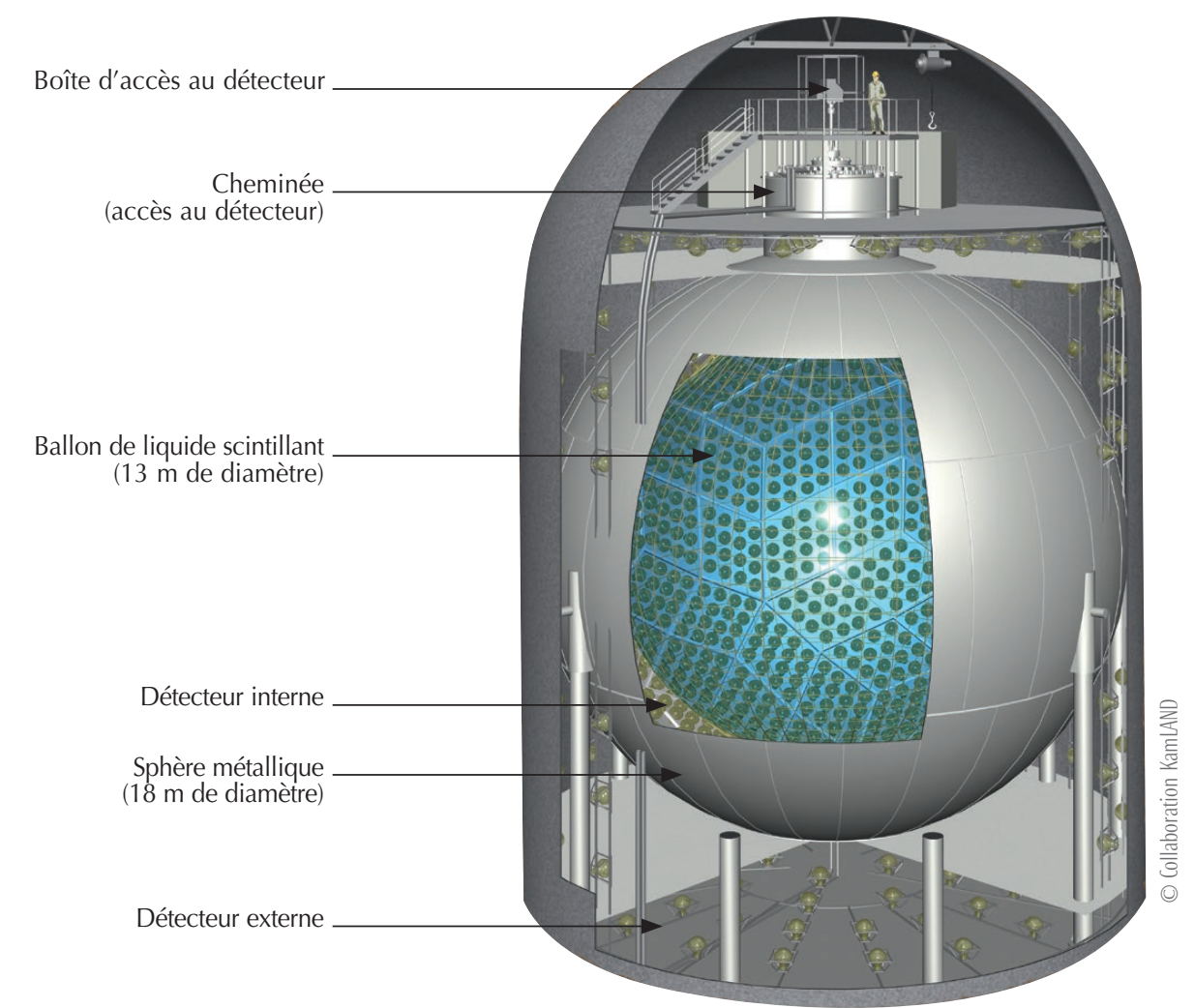

1. Le détecteur KamLAND, situé dans la mine de Kamioka au Japon. La partie la plus sensible du détecteur, le ballon de liquide scintillant, est protégée des événements parasites de bruit de fond extérieurs par les couches d'huile et d'eau qui l'entourent. Afin de pouvoir être sensible au minuscule flash de lumière émis par l'interaction d'un neutrino, chaque composant du détecteur doit être analysé et doit respecter des normes très strictes de compatibilité chimique, de radioactivité et de propreté. Le seul accès au détecteur s'effectue par le haut dans une boîte continuellement sous atmosphère protectrice d'azote. (www.kamland.stanford.edu/Pictures/Pictures.html)

ans de la part des deux expériences parmi les plus sensibles au monde : KamLAND au Japon et Borexino.

De conceptions similaires, ces deux détecteurs à liquide scintillant se différencient par leurs tailles et les signaux parasites perturbant leurs mesures. Avec 1000 tonnes d'huile scintillante, le détecteur KamLAND est trois fois plus volumineux que Borexino, ce qui augmente significativement son taux de détection. Mais étant situé en plein milieu de l'archipel japonais, KamLAND fut, jusqu'en 2011, date de l'accident nucléaire de Fukushima et de l'arrêt de la production nucléaire au Japon, sujet à un important flux de neutrinos de réacteurs. Or, bien qu'à l'origine d'importantes découvertes en physique du neutrino, les neutrinos de réacteurs sont un bruit de fond pour la détection des géoneutrinos, car leur gamme d'énergie s'étend de 0 à $10 \mathrm{MeV}$ et se confond donc en partie avec celle des géoneutrinos (de 0 à 3,3 MeV). D'où l'importance d'évaluer leur contribution avec précision, à défaut de ne pas pouvoir limiter leur nombre en se plaçant à l'écart des réacteurs nucléaires voisins. On notera que les neutrinos solaires ainsi que les neutrinos atmosphériques issus $\mathrm{du}$ rayonnement cosmique ne contribuent pas au bruit de fond pour la détection des géoneutrinos. En effet, les neutrinos solaires sont des neutrinos électroniques et non pas des antineutrinos électroniques, et ne peuvent pas interagir par désintégration bêta inverse. Les neutrinos atmosphériques, majoritairement de saveur muonique et donc également non sensibles à la désintégration bêta inverse, ont une énergie moyenne d'environ $1000 \mathrm{MeV}$, mille fois supérieure à celle des géoneutrinos.

\section{Résultats actuels}

C'est donc en partie grâce à sa position géographique, située à plus de $500 \mathrm{~km}$ des réacteurs nucléaires de la vallée du Rhône, que le détecteur Borexino a rapporté en juin 2015 la détection de 24 candidats géoneutrinos après un temps de mesure d'environ 2000 jours [3]. La probabilité que ce résultat soit purement dû au hasard étant de 1 sur 300 millions, il s'agit donc d'une découverte, signant ainsi la fin de l'ère de recherche des géoneutrinos et le début de celle de leur utilisation pour la physique des particules et la géophysique. Ce résultat permet déjà d'obtenir des informations sur la chaleur émise par la 
Terre en se basant sur des modèles géologiques existants, extrapolés à partir de mesures et de sondages. En supposant une masse totale de thorium quatre fois supérieure à celle de l'uranium, la chaleur totale émise par ces deux chaînes de désintégration serait comprise entre 11 et $52 \mathrm{TW}$. Si on estime que la masse totale de potassium est dix mille fois supérieure à celle de l'uranium, la chaleur radiogénique émise par ces trois radioéléments serait de $33_{-20}^{+28}$ TW, un résultat en accord avec la valeur de $47 \pm 2$ TW citée plus tôt. Il est encore trop tôt pour émettre une hypothèse, même avec un niveau de confiance faible, sur le meilleur modèle BSE par rapport à un autre. La marge d'erreur sur les mesures étant directement liée aux nombres de géoneutrinos détectés, il est certain que des résultats de plus en plus précis seront annoncés prochainement.

\section{Perspectives}

Maintenant que leur existence est formellement établie, les géoneutrinos sont devenus un signal à part entière et leur détection est l'un des objectifs principaux de tous les gros détecteurs à liquide scintillant, en opération ou futurs. Alors que Borexino et KamLAND continuent à accumuler des données, le détecteur $\mathrm{SNO}+$ au Canada (voir la photo de la p. 11) vient d'entrer en service et, grâce à son faible taux de bruit de fond et à sa forte taille (néanmoins légèrement inférieure à celle de KamLAND), apportera prochainement une troisième mesure du flux de géoneutrinos. Cette troisième mesure est très attendue par les géophysiciens, car elle permettra de mieux évaluer les concentrations en radioéléments de la croûte et du manteau à différents endroits du globe terrestre. En effet, la composition de la croûte n'est pas identique sur chaque plaque ni en chaque position de celle-ci, ce qui entraîne des disparités de flux de géoneutrinos observés (fig. 2).

Les détecteurs Borexino, KamLAND et $\mathrm{SNO}+$ offriront aux physiciens trois mesures différentes, mais ils auront en commun le fait que les flux de géoneutrinos mesurés proviendront à la fois de la croûte et du manteau terrestres, sans possibilité de distinguer les deux. Or il sera crucial d'isoler la fraction de géoneutrinos venant du manteau afin de raffiner les modèles de la dynamique interne de notre Terre, une

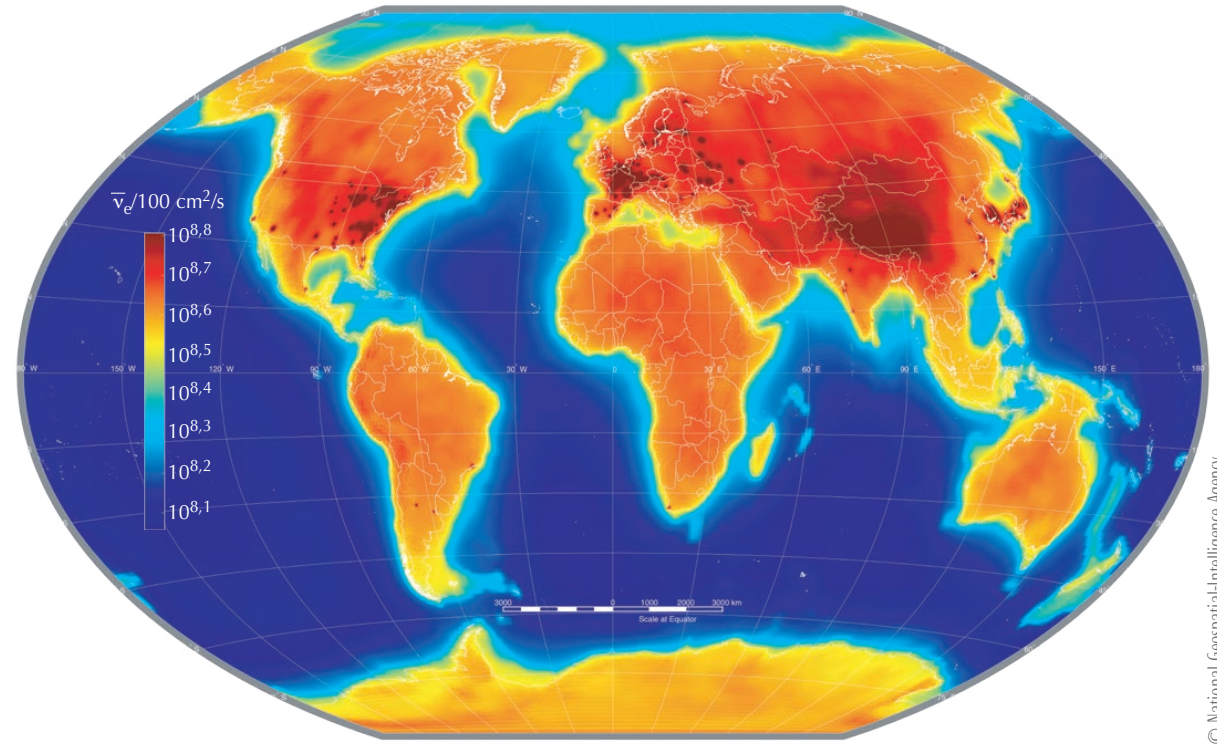

2. Modélisation du flux d'antineutrinos à la surface du globe. Seuls les antineutrinos de réacteurs et les géoneutrinos provenant des différentes couches terrestres sont pris en compte. L'échelle de couleur indique le nombre d'antineutrinos par $100 \mathrm{~cm}^{2}$ et par seconde. Les points intenses (de couleur rouge foncé) observés en Europe, en Amérique du Nord et en Asie de l'Est représentent les réacteurs nucléaires en activité. www.nga.mil/MediaRoom/PressReleases/Pages/Antineutrino.aspx.

détermination difficilement atteignable par les moyens actuels tels que les sondages géologiques. En utilisant les résultats de récentes modélisations de la croûte proche du détecteur, les résultats de Borexino permettent de rejeter l'hypothèse que tous les géoneutrinos détectés proviennent de la croûte seule avec une certitude de $98 \%$, malheureusement insuffisante pour être considérée officiellement comme une découverte.

Il faudra donc installer un détecteur près du plancher océanique et loin des côtes, afin de s'affranchir du signal de l'épaisse croûte continentale. Ce sera l'objectif de la prochaine génération de détecteurs de neutrinos à liquide scintillant.

Le projet HanoHano, un projet de détecteur sous-marin de plus de 10000 tonnes, déployé au large de l'archipel d'Hawaï, a été proposé. Outre sa taille imposante, ce détecteur bénéficierait d'un des taux de bruit de fond les plus bas imaginables, grâce à son principe de déploiement qui lui permettrait d'être protégé du rayonnement cosmique par plusieurs kilomètres d'eau, mais également grâce à sa position géographique, éloignée de tout réacteur nucléaire. En plus d'être un détecteur polyvalent pour la physique du neutrino, HanoHano, installé sur la croûte océanique épaisse de seulement $8 \mathrm{~km}$, serait donc tout particulièrement sensible aux géoneutrinos du manteau terrestre, contrairement aux autres détecteurs qui sont situés sur la croûte continentale, épaisse d'environ $35 \mathrm{~km}$.

À plus courte échéance, le projet Jinping en Chine, d'une construction similaire aux trois détecteurs existants et d'une masse de 4000 tonnes, est en cours d'étude et pourrait voir le jour dans les années à venir, offrant une quatrième mesure du flux de géoneutrinos à un endroit où ce flux est dominé par la croûte continentale.

Imaginée dans les années 1960, la détection des géoneutrinos s'est concrétisée en juin 2015 et est maintenant en passe d'être utilisée pour contraindre les modèles de notre Terre [4, 5]. L'élusif neutrino va ainsi dans les années à venir être le maillon reliant deux domaines de la science autrefois distants, la physique des particules et la géophysique. I

\section{Références}

1 - S. Lavignac et M. Zito, Reflets de la physique n ${ }^{\circ} \mathbf{5 0}$ (2016) 4-9.

2• L. Ludhova et S. Zavatarelli, Advances in High Energy Physics 2013 (2013) ID 425693.

3• M. Agostini et al. (Borexino collaboration), Phys. Rev. D 92 (2015) 031101.

4• T. Lasserre, « L'exploration souterraine des cieux", l'Astronomie 121 (juillet-août 2007) 334-342.

5• T. Lasserre et P. Pajot, « L'intérieur de la Terre en direct », La Recherche 401 (octobre 2006) 50-53. 